\title{
Inelastic scattering in ocean water and its impact on trace gas retrievals from satellite data
}

\author{
M. Vountas, A. Richter, F. Wittrock, and J. P. Burrows \\ Institute for Environmental Physics, University of Bremen, Bremen, Germany \\ Received: 4 April 2003 - Published in Atmos. Chem. Phys. Discuss.: 2 June 2003 \\ Revised: 29 August 2003 - Accepted: 4 September 2003 - Published: 15 September 2003
}

\begin{abstract}
Over clear ocean waters, photons scattered within the water body contribute significantly to the upwelling flux. In addition to elastic scattering, inelastic Vibrational Raman Scattering (VRS) by liquid water is also playing a role and can have a strong impact on the spectral distribution of the outgoing radiance. Under clear-sky conditions, VRS has an influence on trace gas retrievals from space-borne measurements of the backscattered radiance such as from e.g. GOME (Global Ozone Monitoring Experiment). The effect is particularly important for geo-locations with small solar zenith angles and over waters with low chlorophyll concentration.
\end{abstract}

In this study, a simple ocean reflectance model (Sathyendranath and Platt, 1998) accounting for VRS has been incorporated into a radiative transfer model. The model has been validated by comparison with measurements from a swimming-pool experiment dedicated to detect the effect of scattering within water on the outgoing radiation and also with selected data sets from GOME. The comparisons show good agreement between experimental and model data and highlight the important role of VRS.

To evaluate the impact of VRS on trace gas retrieval, a sensitivity study was performed on synthetic data. If VRS is neglected in the data analysis, errors of more than $30 \%$ are introduced for the slant column $(S C)$ of $\mathrm{BrO}$ over clear ocean scenarios. Exemplarily DOAS retrievals of $\mathrm{BrO}$ from real GOME measurements including and excluding a VRS compensation led to comparable results as in the sensitivity study, but with somewhat smaller differences between the two analyses.

The results of this work suggest, that DOAS retrievals of atmospheric trace species from measurements of nadir viewing space-borne instruments have to take VRS scattering into account over waters with low chlorophyll concentrations, and that a simple correction term is enough to reduce the errors to an acceptable level.

Correspondence to: $\mathrm{M}$. Vountas

(vountas@iup.physik.uni-bremen.de)

\section{Introduction}

Backscattered light from waters with low concentrations of chlorophyll and gelbstoff - which are commonly referred to as oligotrophic waters - is significantly influenced by Vibrational Raman Scattering (VRS) within the water (e.g. Stavn and Weidemann, 1988; Marshall and Smith, 1990; Haltrin and Kattawar, 1993; Sathyendranath and Platt, 1998; Vasilkov et al., 2002b).

As inelastic scattering redistributes photons over wavelength, VRS fills-in solar Fraunhofer lines, as well as trace gas absorption lines and therefore changes the spectral distribution of scattered light in the atmosphere. When measuring the backscattered radiance from space, this leads to stable spectral features over areas of the world's oceans. This is of particular importance for remote sensing from space as oligotrophic conditions prevail across large parts of the open ocean.

The impact of VRS on backscattered light is comparable to an atmospheric process, known as the Ring effect (Grainger and Ring, 1962). The origin of the Ring effect was found by several independent observations to be Rotational Raman Scattering (RRS) by $\mathrm{O}_{2}$ and $\mathrm{N}_{2}$ (e.g. Kattawar et al., 1981; Vountas et al., 1998, and references therein).

The effect of photon redistribution based on RRS influences atmospheric trace gas retrievals from measurements of backscattered light with space-borne instrumentation significantly (Vountas et al., 1998). Even if the contribution to the filling-in of redistributed photons by VRS in liquid water is usually smaller than the one by RRS in air it still has to be evaluated how large the impact on trace gas retrievals is.

Retrievals from data of the Global Ozone Monitoring Experiment (GOME) are potentially influenced by VRS. The GOME instrument was launched on ERS-2 in 1995 (Burrows et al., 1999). It is a nadir-viewing spectrometer taking backscatter measurements at moderate spectral resolution in the UV and visible spectral range. Global coverage is obtained in three days at the equator; the nominal swath is $960 \mathrm{~km}$ comprising three forward scans leading to a ground pixel size of $320 \mathrm{~km}$ (across track) x $40 \mathrm{~km}$ (along track). 
In a recent paper by Vasilkov et al. (2002b) it was shown that the effect of VRS is most pronounced in the wavelength range between about 340 to $400 \mathrm{~nm}$ for oligotrophic waters. They were the first to show that VRS can have a significant impact on measurements of backscattered light from spaceborne instrumentation. They showed good agreement between model results and GOME data and plan to use the resulting high-frequency spectral structures for chlorophyll retrieval.

In this study we incorporated a simple analytic ocean reflectance model (developed by Sathyendranath and Platt, 1998) in an atmospheric radiative transfer model (Rozanov et al., 1997; Vountas, 1998; Vountas et al., 1998; de Beek et al., 2001) to simulate accurately the upwelling radiance including the effect of VRS.

A first model verification was then performed by running the model for various scenarios (see Sect. 4) and comparing the results visually with those from Vasilkov et al. (2002b).

The model results are then validated using two approaches: (i) against spectra that have been measured by directing one of our ground-based spectrometers directly to fresh water in a swimming pool (Sect. 5) and comparing upand downwelling radiances; (ii) using GOME data measured over oligotrophic oceanic waters (Sect. 6).

Having successfully validated the model and its assumptions a sensitivity study is performed in order to quantify the isolated, theoretical error expected from neglecting the effects of VRS in the retrieval of trace gases using the Differential Optical Absorption Spectroscopy (DOAS) technique. Finally we evaluate a number of selected GOME measurements and apply a VRS compensation spectrum (scheme) in order to estimate the impact of VRS on experimental data.

\section{Historical background}

This study was originally motivated by tests performed from one of us (F. Wittrock) on optimizing the wavelength window size for the DOAS-retrieval of OClO (Wittrock et al., 2000). Larger window sizes ( $357 \mathrm{~nm}$ towards $390 \mathrm{~nm}$ ) led to large and stable fit residuals over oligotrophic waters that had a negative impact on the retrieval. Biologically more active regions of the oceans showed the effect to a much lesser degree as well as cloudy pixels that were not affected. Further tests with fresh water pixels from GOME (the great lakes in north America) also did not lead to a strong residual.

Trace gas retrieval of GOME data typically uses the DOAS method (Platt and Perner, 1980), whereby slant columns of atmospheric trace gases are fitted (and depending on the targeted species converted to vertical columns using a simulation of the Air Mass Factor).

The upper map in Fig. 1 shows results from DOAS retrievals of GOME data over a period of one month (February) in 1999. All known trace gas absorptions and a Ring reference spectrum (Vountas et al., 1998) were fitted in the wave- length range of 345-385 $\mathrm{nm}$. The upper map shows colorcoded $\chi^{2}$-values. High $\chi^{2}$-values indicate a poor fit quality. Comparing geographical regions with high $\chi^{2}$-values and areas having low chlorophyll-a concentrations derived from SeaWiFS data of the same month in 1999 (map below) show good qualitative agreement.

We concluded that the reason for this residual could be due to water-inherent characteristics. However, these characteristics must have been modulated with the Inherent Optical Properties (IOP) of natural waters, so that water rich in chlorophyll-a concentrations would lead to other residuals than oligotrophic water. Comparison of the regions with poor fitting results with those areas, where liquid water absorption can be detected in GOME measurements in the visible spectral region (Richter and Burrows, 2000) also suggested a link between average photon path in ocean water and the observed spectral structures.

In order to test this assumption, we set up an experiment pointing a spectrometer alternatively to the blue zenith-sky and the water of a clear fresh water swimming pool. From the two measurements, a residual was retrieved that was similar to that observed in the GOME data over clear water, demonstrating that the effect was indeed related to scattering on or in the water. The publication of Haltrin and Kattawar (1993); Sathyendranath and Platt (1998) and later Vasilkov et al. (2002b) pointed out that Raman scattering cannot be neglected in oligotrophic waters and leads to filling-in of solar Fraunhofer lines which could lead to effects as the one observed in our experiment. We therefore modeled the impact of Raman scattering on backscattered spectra and studied the resulting changes in the retrieved trace gas columns.

\section{Modeling}

The primary objective of both GOME and SCIAMACHY (Scanning Imaging Absorption Spectrometer for Atmospheric CHartographY) as well as forthcoming GOME-2 is to determine the abundances of atmospheric trace gases, such as $\mathrm{O}_{3}, \mathrm{NO}_{2}, \mathrm{BrO}, \mathrm{OClO}, \mathrm{SO}_{2}, \mathrm{HCHO}, \mathrm{CH}_{4}, \mathrm{CO}$, and $\mathrm{N}_{2} \mathrm{O}$. All these trace gases have narrow band absorptions, which can be utilized in the DOAS approach to derive their effective columns from the atmospheric optical depth. The main advantage of the DOAS retrieval is its high computational speed and the simplicity of the retrieval. Therefore, a possible correction of the effect of VRS on the data should also be simple and preferably have the form of a pseudo-absorber to be included in the fit. This approach is usually taken for the correction of atmospheric Raman scattering, and will here be employed for VRS as well.

Provided a DOAS-type retrieval a Differential Optical Depth (DOD) of the type DOD $=\ln \left(I / I_{0}\right)-P$, with $I$ being the backscattered radiance, $I_{0}$ the extraterrestrial irradiance and $P$ a polynomial, is evaluated. From this formalism 

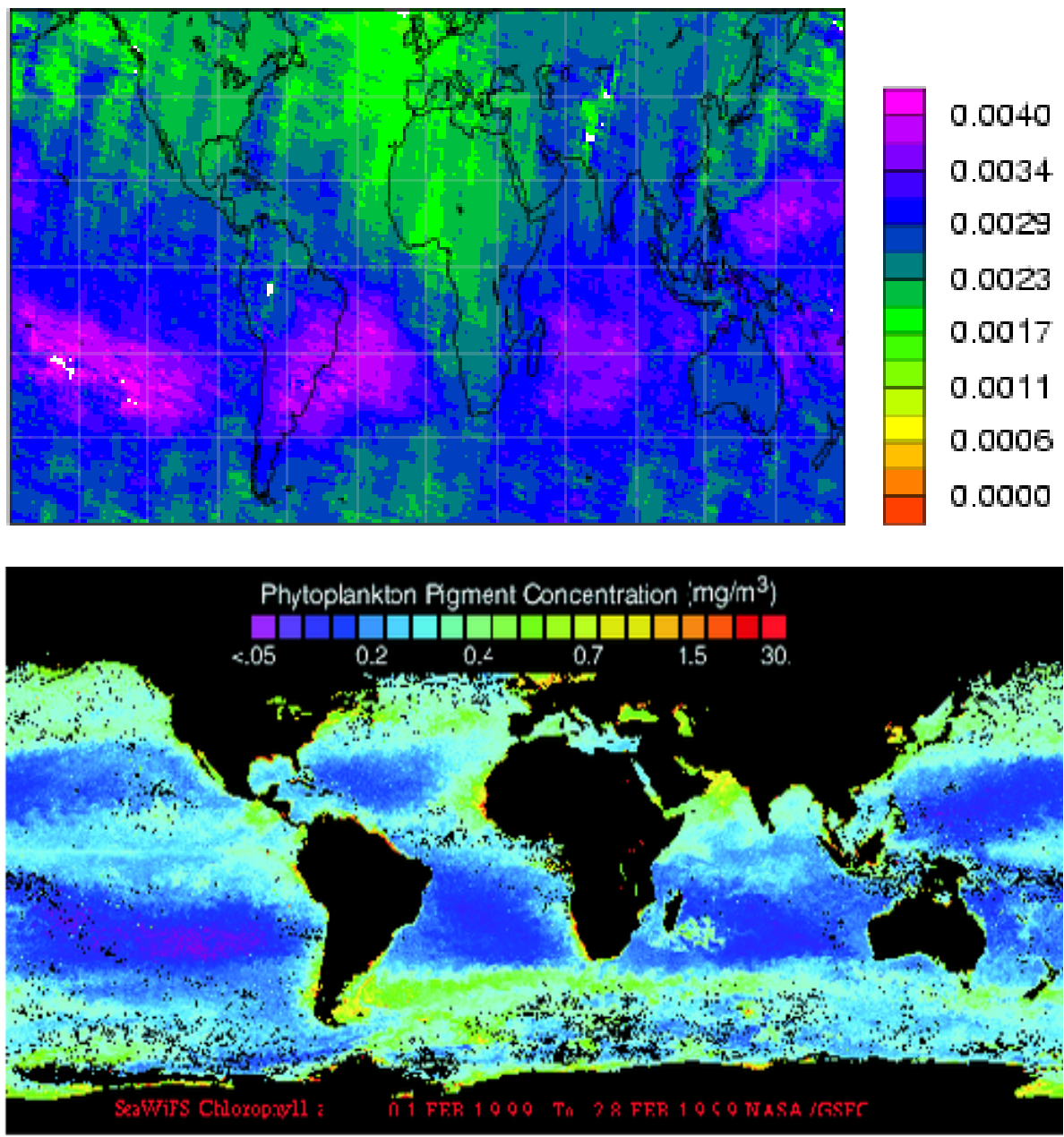

Fig. 1. Above: GOME fit quality results plotted for February 1999; below: Chlorophyll-a concentration from SeaWiFS for the same period.

we define a reference spectrum $r_{r}$ accounting for RRS at a wavelength $\lambda$ as:

$r_{r}(\lambda)=\ln \frac{I^{+\operatorname{RRS}}(\lambda)}{I^{-\operatorname{RRS}}(\lambda)}$.

With $I^{+ \text {RRS }}$ being the modeled radiance taking into account RRS and $I^{- \text {RRS }}$ neglecting it (Vountas et al., 1998). Analogously we define a reference spectrum $r_{w}$ accounting for VRS in ocean water at a wavelength $\lambda$ :

$r_{w}(\lambda)=\ln \frac{I^{+\operatorname{VRS}}(\lambda)}{I^{-\operatorname{VRS}}(\lambda)}$.

In the following $r_{w}$ is also called VRS compensation spectrum. $I^{+\mathrm{VRS}}$ is the modeled radiance taking into account VRS and $I^{- \text {VRS }}$ the radiance neglecting it.

A prerequisite for taking into account VRS in DOAS-type retrievals is therefore a precise knowledge of the quantities $I^{+ \text {VRS }}$ and $I^{- \text {VRS }}$. This requires a coupled atmosphereocean radiative transport model, which is described in this section.
The oceanic model has been incorporated into an atmospheric model by simply passing the reflectance function for a given chlorophyll concentration to the atmospheric model which computes the radiance or flux for a given measurement geometry and atmospheric scenario.

The analytic oceanic reflectance model is based on the work of Sathyendranath and Platt (1998). They found excellent agreement between results generated with their model and those of a relatively complex Monte-Carlo model. Despite this good agreement it should be noted that the model is based on several assumptions and simplifications: (i) the ocean surface is considered to be flat and the water column optically deep, i.e. no bottom effects have been taken into account; (ii) the IOP and mean cosines of the water are distributed uniformly in the water column; (iii) VRS contributes to the wavelength of interest through various source wavelengths: nevertheless all IOPs are computed for one representative source wavelength (see below for further discussion); (iv) The mean cosines are fixed to constant values (Vasilkov et al., 2002b); (v) only first order VRS is computed. 
The result is a wavelength dependent reflectance function that is based on a bio-optical model having only the chlorophyll concentration as a one-parametric input. This assumption is valid whenever the concentration of phytoplankton is significantly larger than non-biogenic particles. Such waters are also referred to as case I waters (Morel and Prieur, 1977).

\subsection{Oceanic model}

The approach is based on seawater reflectance accounting for absorption and elastic, as well as inelastic (transspectral) scattering. Elastic scattering in water is dominated by Rayleigh scattering (or better "Einstein-Smoluchowski" scattering, referring to Mobley (1994) citing Young (1982)).

We adopt a model for the reflected light in the UV and visible wavelength range originally proposed by Sathyendranath and Platt (1998) based on a modified Quasi-Single-Scattering Approximation (QSSA). The total reflectance $R(\lambda)$ at the sea surface including redistribution by VRS for a wavelength of interest $\lambda$ is defined as:

$R(\lambda)=R^{E}(\lambda)+R^{R}(\lambda) *\left[1+\frac{b_{b}^{E}(\lambda)}{\kappa^{E}(\lambda)}+\frac{2 b_{b}^{E}\left(\lambda^{\prime}\right)}{K\left(\lambda^{\prime}\right)+\kappa^{E}\left(\lambda^{\prime}\right)}\right]$

$\lambda^{\prime}$ is a source wavelength which contributes photons (through Raman scattering) to the actual wavelength $\lambda$. The quantities $R^{E}$ and $R^{R}$ are the reflectances at sea surface attributed to elastic (superscript E) and inelastic, i.e. VRS (superscript $\mathrm{R})$ light scattering (defined below). $b_{b}^{E}$ is the backscattering coefficient for elastic scattering. Adopting the same terminology as Sathyendranath and Platt (1998) we have defined $K$ as the downward vertical attenuation coefficient and $\kappa^{E}$ as the first-order elastic attenuation coefficient to be:

$K\left(\lambda^{\prime}\right)=\frac{a\left(\lambda^{\prime}\right)+b_{b}\left(\lambda^{\prime}\right)}{\mu_{d}} \quad$ and $\quad \kappa^{E}\left(\lambda^{\prime}\right)=\frac{a\left(\lambda^{\prime}\right)+b_{b}^{E}\left(\lambda^{\prime}\right)}{\mu_{u}^{E}}$

Where $\mu_{u}^{E}$ is the mean cosine for the up-welling (elastic scattering only) and $\mu_{d}$ the one for the down-welling irradiance. $a$ is the total absorption coefficient and $b_{b}$ is the total backscattering coefficient. The reflectance formula part $R^{E}$ is defined as:

$R^{E}(\lambda)=\frac{s^{E}(\lambda) b_{b}^{E}(\lambda)}{\mu_{d}(\lambda)} * \frac{1}{K(\lambda)+\kappa^{E}(\lambda)}$

where $s^{E}$ is the so-called slope which was set to 1 (see original publication). The reflectance attributed to first-order VRS is defined by:

$R^{R}(\lambda)=\frac{E_{d}\left(\lambda^{\prime}\right)}{E_{d}(\lambda)} * \frac{b_{b}^{R}\left(\lambda^{\prime}\right)}{\mu_{d}\left(\lambda^{\prime}\right)} * \frac{1}{K(\lambda)+\kappa^{R}(\lambda)}$.

where $b_{b}^{R}$ is the backscattering coefficient for VRS and the attenuation coefficient for first-order VRS is approximated by $\kappa^{R}=\left(a+b_{b}^{E}\right) / \mu_{u}^{R} . E_{d}\left(\lambda^{\prime}\right)$ and $E_{d}(\lambda)$ are the down-welling irradiance at sea surface for source and actual wavelength $\left(\lambda^{\prime}\right.$, $\lambda)$, respectively.
For Eq. (3) being valid it is necessary to have a vertically homogenous water body. The quantities required for the computation of $R(\lambda)$ are the apparent optical properties (AOP) $\mu_{d}, \mu_{u}^{R}$ and $\mu_{u}$ which were set $\mu_{d} \approx 0.75$ (Sathyendranath and Platt, 1998) and $\mu_{u}=\mu_{u}^{R} \approx 0.5$ (Vasilkov et al., 2002b). The determination of the down-welling flux $E_{d}$ is done using SCIATRAN, the radiative transfer model described in Sect. 3.3.

The inherent optical properties, $a, b_{b}^{E}$ and $b_{b}^{R}$ and the corresponding wavelengths are discussed in Sect. 3.2.

\subsection{Inherent optical properties of sea water}

Apparent optical properties such as the reflectance as given by Eq. (3) require the knowledge of inherent optical properties (IOP) of the water body. With the exception of the VRS (back) scattering coefficient $b^{R}$ which is determined through a simple exponential expression as a function of $\lambda^{\prime}$ both IOPs $a$ and $b_{b}$ will depend only on chlorophyll concentration $C$ $\left[\mathrm{mg} / \mathrm{m}^{3}\right]$. This section describes how to determine all three quantities (IOPs). It should however be noted that measurements and corresponding parametrizations of IOPs in the UV are rare and often unreliable (Vasilkov et al., 2002a). Accordingly, errors in the IOPs will translate into any estimate of the impact of VRS on trace gas retrievals. However, quantification of the translation of IOP related errors into impact of VRS on trace gas retrieval is beyond the scope of this paper.

The influence of VRS on GOME measurements is largest in case I water regions, where all inherent optical properties, except those of water itself, depend on phytoplankton concentration $C$. For estuarine and coastal waters (case II) the simple relationships found in case I waters do not hold and the contribution of detrital and dissolved organic matter (DOM) components to $a$ is significantly larger than the one of phytoplankton.

\section{Total Absorption Coefficient:}

The total absorption coefficient $a$ of a sea water body can be expressed as follows:

$a(\lambda, C)=a_{w}(\lambda)+a_{C}(\lambda)+a_{\mathrm{DOM}}(\lambda)$.

Pure water absorption coefficients $a_{w}$ were taken from spectral interpolation over two data sets (Quickenden and Irvin, 1980; Pope and Fry, 1997) as proposed by Vasilkov et al. (2002b).

$a_{C}$ is the chlorophyll-related absorption coefficient taken from Vasilkov et al. (2002a) (data sets from personal communication).

According to the model of Morel (1988) the DOM absorption at $440 \mathrm{~nm}$ is $20 \%$ of the total absorption of pure seawater and particulate matter and an exponential function was used to describe the spectral variation of $a_{\mathrm{DOM}}$ :

$a_{\mathrm{DOM}}=0.2 *\left[a_{w}(440)+a_{C}(440)\right] * e^{-S *(\lambda-440)}$ 
The spectral slope $S$ of DOM was set to the wide-spread value of $0.014 \mathrm{~nm}^{-1}$ which, however is controversially discussed in literature (Vasilkov et al., 2002a; Patterson, 2000).

\section{Total Elastic Backscattering Coefficient:}

In order to compute Eq. (3) the total elastic backscattering coefficient $b_{b}^{E}$ must also be known. A bio-optical model for this spectral quantity can be found in Morel (1988):

$$
\begin{aligned}
b_{b}(\lambda)= & 0.5 b_{w}(\lambda)+ \\
& {\left[0.002+0.02(0.5-0.25 \log C) \frac{550}{\lambda}\right] * } \\
& {\left[0.3 C^{0.62}-b_{w}(550)\right] . }
\end{aligned}
$$

The pure sea water scattering coefficients $b_{w}$ were taken from Smith and Baker (1981).

\section{Backscattering Coefficients for VRS:}

The mean wavenumber shift of $3357 \mathrm{~cm}^{-1}$ represents two fundamental $\mathrm{OH}$ stretch vibration modes of the water molecule that are further modified by hydrogen bonding and rotational fine structure. These interactions induce a broad band of emissions around the mean wavenumber shift so that water Raman emissions occur over a band of $\approx 30-50 \mathrm{~nm}$. Walrafen (1967) showed that the spectral shape of measurements of Raman scattered intensities of pure water could be well reproduced by four different Gaussian functions. This empirical approach for calculating Raman scattering crosssections was adopted by several authors devoted to this topic, for example Kattawar and Xu (1992) and Haltrin and Kattawar (1993). We have adapted the determination of the source wavelengths $\lambda^{\prime}$ from Haltrin and Kattawar (1993).

The values for the scattering coefficient $b^{R}$ were determined using the following commonly used relation:

$b^{R}\left(\lambda^{\prime}, \lambda\right)=b_{0}^{R}\left(\lambda^{\prime} / \lambda_{0}\right)^{P}$,

where $b^{R}$ is the Raman scattering coefficient at wavelength $\lambda^{\prime}$ and $b_{0}^{R}=2.610^{-4} \mathrm{~m}^{-1}$ is the Raman absorption coefficient at wavelength $\lambda_{0}=488 \mathrm{~nm}$. Bartlett et al. (1998) showed that the exponent $P$ is near to 5.3 (instead of 5 as shown in older studies).

The phase function VRS is nearly isotropic (Ge etal., 1993) and leads to the simple relation between scattering and backscattering coefficient for VRS $b_{b}^{R}=0.5 b^{R}$ (required in Eq. 4).

\subsection{Atmospheric model}

Within the framework of this study the atmospheric model is necessary to provide the base for the incorporation of the oceanic reflectance function accounting for VRS. It is furthermore necessary to determine the a priori unknown ratio of the down-welling irradiance $E_{d}$ at $\lambda$ and $\lambda^{\prime}$ as required in Eq. (4). From a formal point of view the reflectance (ocean)

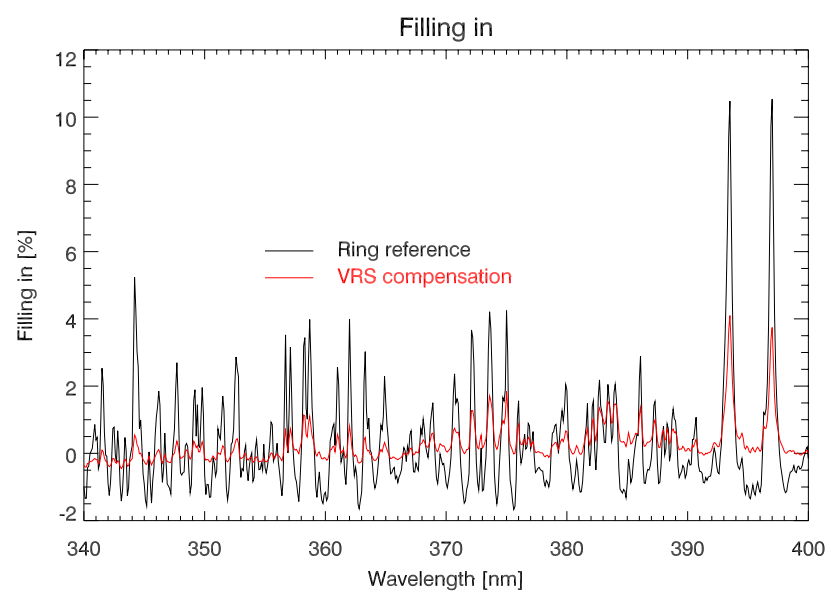

Fig. 2. Comparison of Ring reference and VRS compensation spectrum as defined in Eq. (2).

model provides the lower boundary condition of the atmospheric model.

The radiative transfer model used is SCIATRAN (formerly known as GOMETRAN) (Rozanov et al., 1997). The solution of the radiative transfer equation computed by SCIATRAN is based on the finite difference method and has been successfully validated against DISORT. The code calculates the complete unpolarized radiation field and irradiance for ground- or satellite viewing geometry for a given atmospheric composition with respect to the extra-terrestrial flux. The program also calculates appropriate weighting functions which are the response of the radiation field to a change of atmospheric parameters. The mathematical principles of the model are described in detail in Rozanov et al. (1997). The radiative transfer equation is evaluated in plane-parallel geometry, with the exception of the solar source function which is calculated in spherical geometry taking into account refraction. The model was extended to include inelastic scattering by RRS in the atmosphere and has been successfully validated and applied to remote sensing data (Vountas et al., 1998).

\subsection{Notes on Implementation}

Our VRS model neglects the energy transferred from a source wavelength to shorter wavelengths. This assumption goes back to the physics of Raman scattering. AntiStokes-Scattering (scattering from longer to shorter wavelengths, which is an increase of photon-energy after scattering occured) is only possible if the molecule is already in an excited state -and some always are at any temperature above absolute zero. In this case the molecule may emit a photon of shorter wavelength than the incident photon, thereby return to a lower rotational or vibrational state. However, at temperatures of liquid water, Raman scattering from longer to shorter wavelengths is insignificant because too few molecules are in an excited state. 
VRS generates photons at a band of wavelengths due to scattering at a source wavelength $\lambda^{\prime}$. Analogous, more than one source wavelength contributes to the radiation field at a wavelength of interest $\lambda$. As the VRS band is much wider (about $12 \mathrm{~nm}$ at $370 \mathrm{~nm}$ ) than the spectral resolution of the instrument considered here (GOME-resolution is about 0.2 $\mathrm{nm}$ ) the integrated contribution to $\lambda$ is computed.

\section{Model results}

Figure 2 shows the percentage of filling-in in the wavelength range $340-400 \mathrm{~nm}$. The filling-in was defined by $100 *\left(I^{+}-I^{-}\right) / I^{-}$. Both spectra were prepared with the same atmospheric and geometric setup based on a solar spectrum measured by GOME. The VRS compensation spectrum was computed assuming a chlorophyll concentration of $0.01\left[\mathrm{mg} / \mathrm{m}^{3}\right]$. The solar zenith angle was set to $32^{\circ}$ and a maritime Lowtran aerosol scenario (Kneizys et al., 1986) has been selected. The vertical distribution of trace gases, temperature and pressure was taken from the MPI climatology (Brühl and Crutzen, 1992) for October.

The Ring reference spectrum shows larger absolute spectral dynamics than to the VRS compensation spectrum. We have checked the spectral correlation between $r_{w}$ and $r_{r}$ by using a model approach: both spectra have been modeled for identical atmospheric and oceanic conditions and were fitted to each other in a DOAS-like fashion. In spite of a reasonable overall fit result there are still enough remaining differences to enable independent fitting of both spectra. This is a result of the different efficiencies of the processes and a much smaller spectral redistribution range of RRS of about \pm 2 $3 \mathrm{~nm}$ in this wavelength region as compared to VRS which has an average shift of about $40 \mathrm{~nm}$. We therefore conclude that the influence of VRS on DOAS retrievals even in small wavelength windows $(<10 \mathrm{~nm})$ is not negligible because $r_{r}$ cannot fully compensate the filling-in due to VRS (see below in Sect. 6). This fact can potentially be exploited for the determination of chlorophyll concentrations in sea water using DOAS retrievals.

The VRS spectrum is in good qualitative agreement with the results presented by Vasilkov et al. (2002b). However, they prepared their spectra on the basis of a slightly modified reflectance formula and used a four times lower spectral resolution as an input for their model. Therefore, a direct comparison has not been performed.

VRS spectra for other chlorophyll values $C$ were also created. The overall behavior was similar to the results of Vasilkov etal. (2002b): The filling-in significantly increases with decreasing chlorophyll-a concentrations. This was expected because $C$ governs the penetration depth and therefore the number of backscattered photons in case I waters. Vasilkov et al. (2002b) concluded from the strong interrelation between $C$ and the magnitude of filling-in that chlorophyll-a concentrations can be retrieved from spectra of the backscattered light measured from satellite.
In an additional test, (fair weather) clouds were added to the SCIATRAN simulation. As a result, the effect reduces by three orders of magnitude for satellite geometry and can therefore be neglected. This is not surprising, as even optically thin clouds reflect a large part of the incoming light and therefore dramatically reduce the impact of up-welling radiation from water on space borne observations.

\section{Swimming pool experiment}

Historically the authors' assumptions on the origin of the residuals observed in GOME data was based on circumstantial evidence. The effect was not clearly identified and an instrumental artifact could not fully be excluded. In order to isolate the source of the observed structures, we have in spring 1999 performed measurements on the ground in Bremen, $53^{\circ} \mathrm{N}$ with one of our grating spectrometers usually employed for zenith-sky measurements. The spectral resolution of the instrument is comparable to the one of GOME in the UV channels; however, the spectral range covered is only 344-388 nm.

The instrument was set up on top of a $10 \mathrm{~m}$ high platform over the basin of a swimming pool that had been filled with fresh chlorinated water the day before. Light was fed into the spectrometer using a depolarizing quartz-fibre bundle attached to a small telescope with a narrow field of view $\left(<1^{\circ}\right)$. The telescope was pointed alternatively to the blue sky and to the water surface which was shaded from direct sun in the field of view of the telescope to avoid interference from sun glint. During part of the measurements, a rotatable polarizer was mounted in the telescope and measurements were taken alternating between measurements in parallel and perpendicular polarization (with respect to the solar azimuth).

The sky was clear during the measurement period with some evidence for enhanced aerosol loading from the brightness of the horizon. The water in the basin was very clear and both the blue bottom and the sides of the basin were clearly visible indicating that at least in the visible spectral range, border effects can not be excluded.

When analyzing the results of the crossed-polarizer measurements in the way proposed by Solomon et al. (1987), the zenith-sky data produced a nice spectral Ring effect signature as expected for Rotational Raman scattered light. However, the nadir viewing measurements did not show such an effect, and very little difference was observed between the two polarisation directions. However, when upwards and downwards looking measurements were compared, a clear signature showed up that after subtraction of a polynomial was very similar to the residual structures observed in the GOME measurements over clear ocean areas. We therefore concluded, that the observed features are not an instrumental artefact of GOME, but rather the result of a scattering or absorption effect of sun light in liquid water. 

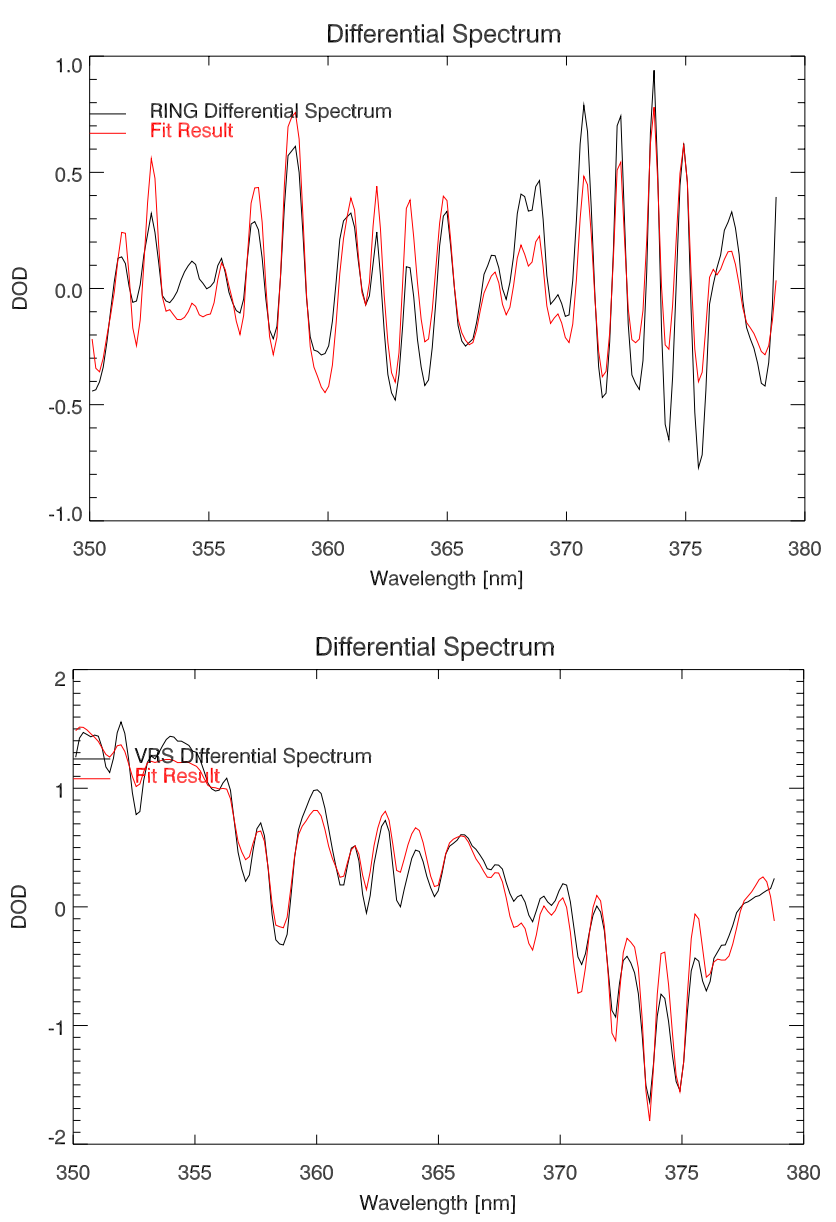

Fig. 3. Fitting of Swimming pool data. Above: Fit results for Ring reference spectrum; below: Fit results for VRS compensation spectrum.

As a next step, we tried to simulate the swimming pool measurements with the VRS model developed in this study. The approach was to analyze the measured spectra using a simple DOAS approach and fitting an accurately modeled Ring $r_{r}$ and VRS compensation $r_{w}$ spectrum to the data.

$r_{r}$ and $r_{w}$ were computed according to the definitions in Eqs. (1) and (2) for an extremely low chlorophyll concentration $\left(0.0001\left[\mathrm{mg} / \mathrm{m}^{3}\right]\right)$. SCIATRAN was set up in a way that the conditions prevailing during the experiment could be taken into account as accurate as possible. $I^{ \pm \mathrm{RRS}}$ and $I^{ \pm \text {VRS }}$ were computed with a highly sampled and resolved solar spectrum (Kurucz et al., 1984). The spectra were then smoothed and re-sampled to the instrument resolution and spectral spacing (full width at half maximum was $0.5 \mathrm{~nm}$ and average sampling was $0.16 \mathrm{~nm}$ ).

Figure 3 shows the comparison of the fitted result for both spectra - VRS and RRS - and the corresponding residuals of the experimental spectrum. The difference between the fitted result and corresponding residual is identical to the overall residual.
The agreement of modeled and fitted Ring spectrum $r_{r}$ is slightly poorer than that of the VRS compensation spectrum $r_{w}$. The reasons for this behavior are still not fully understood. However, the overall agreement especially in case of the comparison of $r_{w}$ is very good suggesting that the fillingin of Fraunhofer lines by VRS in liquid water is adequately modeled.

From the swimming pool experiment and the model calculations we conclude, that (i) VRS can have a significant impact on backscatter experiments and (ii) that our model can adequately model the effect of VRS on upwelling radiation over water surfaces in the UV. The next step was to analyze if the VRS compensation spectrum also fits well to the GOME measurements.

\section{GOME}

Both modeled spectra, the VRS compensation and Ring reference spectrum, were used within DOAS retrievals of GOME data in order to show their validity for remote sensing data measured from GOME. Similar results are expected for SCIAMACHY (Scanning Imaging Absorption Spectrometer for Atmospheric CHartographY) once calibrated spectra are available.

Several DOAS fits of clear-sky GOME data measured over the Pacific ocean, near Easter island in February 1999, where low chlorophyll-a concentrations could be expected (see maps in Fig. 1) were performed. A wavelength window between 347 and $385 \mathrm{~nm}$ has been selected. The above described spectra (Ring reference and VRS compensation spectrum) were fitted in addition to the reference spectra of $\mathrm{NO}_{2}$, $\mathrm{O}_{4}, \mathrm{BrO}, \mathrm{O}_{3}$ (two spectra with different temperatures) and an under-sampling correction spectrum.

Small wavelength shifts due to tiny differences in the wavelength scale used in the VRS, Ring, cross-sections, GOME irradiance and radiance spectra are corrected by allowing a non-linear shift-and-squeeze of the wavelength axis for each spectrum relative to an arbitrary reference, here the GOME radiance.

The fit results are shown in Fig. 4. The modeled Ring reference spectrum compared to the rest of the fit (except Ring effect contributions) is shown in the upper plot, the result for the modeled VRS compensation spectrum in the lower plot. Both reference spectra could nicely be identified in the data, indicating that the effects of VRS as predicted by the model are in fact observed in the DOD evaluated. A remaining apparent spectral feature is a tripod at about $383 \mathrm{~nm}$ which can be reproduced neither by the Ring reference nor by the VRS compensation spectrum.

It should be noted that prior to this study DOAS retrievals of measurements potentially influenced by VRS where performed using an empirical approach in order to reduce the impact of VRS. 

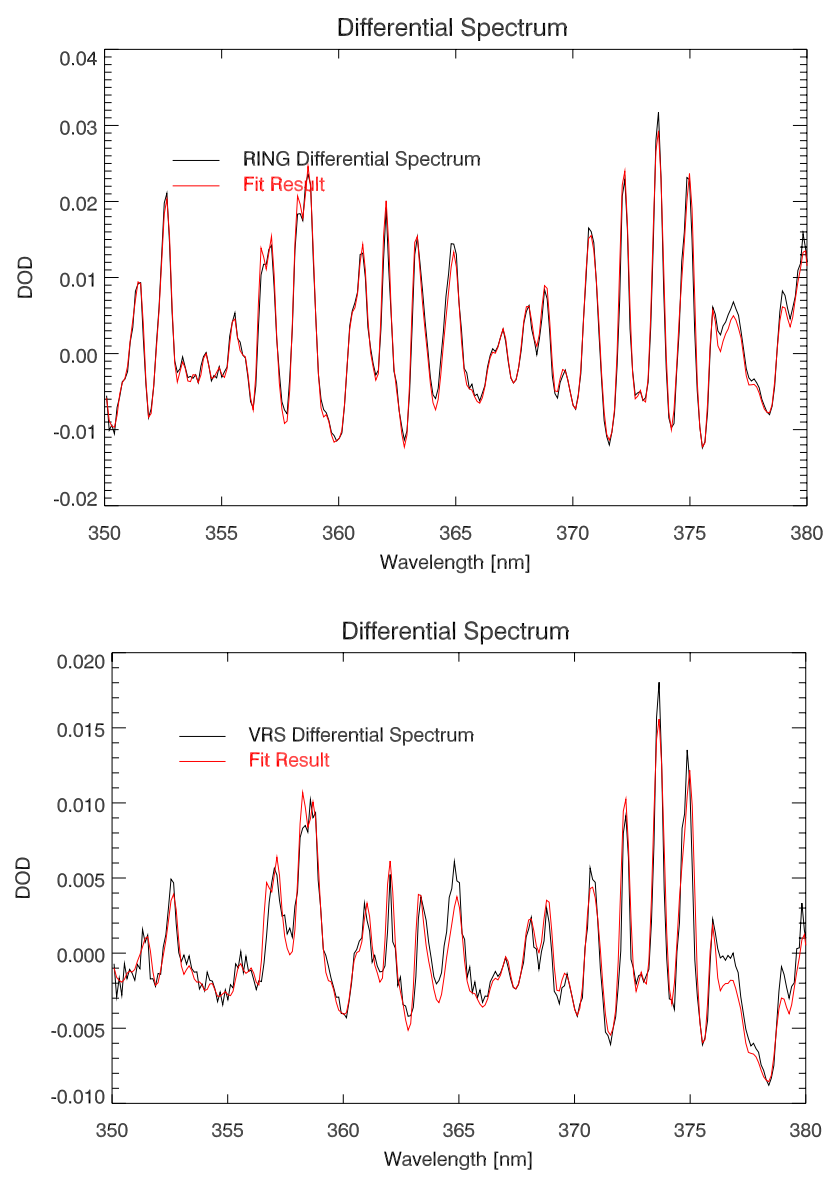

Fig. 4. DOAS fits of clear-sky GOME data (Level 1 filename: 90208175). Above: Fit results for Ring reference spectrum; below: Fit results for the VRS compensation spectrum.

\section{Trace gas retrieval: case studies}

Having demonstrated that VRS signatures are observable in GOME data over clear waters, and that our model provides an adequate simulation of the effect, we have performed sensitivity studies to evaluate the effect of VRS on trace gas retrievals. A mixture of several different trace gas concentrations was used, each appropriate for the selected target species, Bromine Monoxide ( $\mathrm{BrO}$ ). For these scenarios, DODs have been modeled that include the effect of VRS. Using a standard DOAS retrieval, the modeled data have then been analyzed with and without applying a VRS compensation spectrum to determine the impact of VRS on the DOAS retrieval of the trace gas columns.

These tests should provide information on the theoretical accuracy if VRS is neglected in DOAS-like trace gas retrievals. For simplicity and clarity, instrumental characteristics have not been taken into account. The strategy involved only a few scenarios which however are representative for real space-borne measurements.

\subsection{Strategy}

Optical Depths $\mathrm{OD}=\ln \left(I / I_{0}\right)$ were computed using various atmospheric scenarios, based on the MPI climatology (Brühl and Crutzen, 1992) for October. However, for BrO the trace gas amount was increased by scaling the whole profile in order to rise the DOD for both gases to values that are routinely retrieved by GOME. Pressure, temperature and vertical profiles of all other trace gases remained unchanged. The solar irradiance $I_{0}$ was taken from Kurucz et al. (1984) and re-sampled to $0.2 \mathrm{~nm}$. The scenarios differed in solar zenith angle (SZA) and chlorophyll concentration $C$ :

$$
\begin{aligned}
& \text { - } \mathrm{SZA}=30,50,70^{\circ} \\
& \text { - } C=0.001,0.01,0.1\left[\mathrm{mg} / \mathrm{m}^{3}\right] .
\end{aligned}
$$

VRS increases with decreasing values of the SZA and C, hence significant differences for the impact of VRS for all scenarios are expected.

Radiances (for OD), VRS compensation spectra and Ring reference spectra were calculated on the same wavelength grid as the solar irradiance. All quantities were computed for all 9 scenario combinations using SCIATRAN. SCIATRAN's input spectra (trace gas absorption spectra etc.) were interpolated to the wavelength grid of the solar irradiance. In addition to molecular scattering processes (RRS and Rayleigh), aerosol scattering was also modeled assuming a maritime Lowtran aerosol scenario (Kneizys et al., 1986).

The evaluation of the OD was performed with the DOAS method using the trace gas reference spectra involved in the previous modeling and various Ring and VRS compensation spectra. ODs for all scenarios were evaluated with the corresponding trace gas spectra, taking

1. adequate RRS and VRS spectra into account to test for consistency and to use the resulting $S C$ as the true reference,

2. an adequate RRS but no VRS spectra into account to show the isolated impact of neglecting VRS in the retrieval.

The first step in this study was to perform test 1 . and to retrieve a $S C$ that served as a correct value $S C_{\text {true }}$ (perfect fit values can be found in the third column in Table 1). The second test led to different values, $S C_{\text {retrieved, }}$, that were ratioed to the correct value in order to define an error, Diff $=100.0^{*}\left(S C_{\text {retrieved }}-S C_{\text {true }}\right) / S C_{\text {true }}$.

For the sake of brevity the results for the neglect of both: RRS and VRS spectrum in the retrieval are not shown. This test showed the largest errors and non-acceptable fit residuals for all target species. All results are listed in Table 1.

In order to retrieve $\mathrm{BrO}$ all quantities for the DOAS evaluation were prepared in a wavelength range slightly larger than the fitting window of $344.7-359 \mathrm{~nm}$ which has been used for the retrieval of $\mathrm{BrO}$ from GOME data (Richter et al., 
Table 1. Slant column fitting results.

\begin{tabular}{|c|c|c|c|c|}
\hline \multicolumn{5}{|c|}{$\begin{array}{l}\text { BrO Slant Column }\left[10^{14} \text { molec. } \mathbf{c m}^{-2}\right] \\
\text { Error=100.0* }\left(S C_{\text {Retrieved }}-S C_{\text {true }}\right) / S C_{\text {true }}\end{array}$} \\
\hline \multirow[b]{2}{*}{$C\left[\mathrm{mg} / \mathrm{m}^{3}\right]$} & \multirow[b]{2}{*}{$\operatorname{SZA}\left[{ }^{\circ}\right]$} & \multirow{2}{*}{$\begin{array}{c}\text { Fitting RRS \& VRS } \\
\text { SC true }^{\text {tris }}\end{array}$} & \multicolumn{2}{|c|}{ Fitting RRS only (neglecting VRS) } \\
\hline & & & $\mathrm{SC}_{\text {Retrieved }}$ & Error [\%] \\
\hline \multirow{3}{*}{0.1} & 30 & 4.407 & 3.395 & -23.0 \\
\hline & 50 & 5.171 & 4.298 & -16.9 \\
\hline & 70 & 7.490 & 6.854 & -8.5 \\
\hline \multirow{3}{*}{0.01} & 30 & 4.441 & 2.941 & -33.8 \\
\hline & 50 & 5.202 & 3.896 & -25.1 \\
\hline & 70 & 7.516 & 6.554 & -12.8 \\
\hline \multirow{3}{*}{0.001} & 30 & 4.450 & 2.848 & -36.0 \\
\hline & 50 & 5.210 & 3.812 & -26.8 \\
\hline & 70 & 7.522 & 6.491 & -13.7 \\
\hline
\end{tabular}

1998). SCIATRAN's model results were calculated including the absorption of $\mathrm{BrO}$, the impact of VRS and the influence from the Ring effect. The BrO SCs computed from the ODs of all chlorophyll variations are very similar.

\subsection{Discussion}

Absolute errors larger than $8 \%$ were found for all scenarios modelled when neglecting the VRS compensation spectrum in the retrieval (Table 1). As anticipated in former sections, a Ring (RRS) spectrum is obviously not capable of compensating for the lack of a VRS compensation spectrum because of the significantly different spectral structure of the processes.

Decreasing the chlorophyll concentration from a typical value for clear ocean waters of 0.1 to extremely low values of 0.01 and $0.001\left[\mathrm{mg} / \mathrm{m}^{3}\right]$ led (for all SZA investigated) to substantial increases of $\mathrm{BrO} S C$ errors. On the other hand, an increase of SZA reduced the error significantly. Both dependencies, the one on $C$ and on SZA, reflect the dominance of VRS for small SZA and small chlorophyll concentrations and its decreasing importance for large SZA and eutrophic waters.

For a fixed SZA and varying values of $C$ a slight change in the slant columns of $\mathrm{BrO}$ can be observed. The reason for this is a change in the number of reflected photons from water surface. With decreasing values of $C$ the ground albedo increases, which results in an increase of the average slant path of the light.

As demonstrated in an earlier publication (Vountas et al., 1998), trace gas retrievals can be significantly biased if the Ring effect is neglected or a wrong Ring reference spectrum is used. This is of special importance for a minor trace gas such as BrO (Burrows et al., 1998). The neglect of RRS lead to errors of over $100 \%$.

Various tests (not shown in the table) using inadequate VRS spectra in the BrO revealed that inaccurate assumptions on VRS still lead to an improvement of the BrO SC. This does not hold for an inadequate RRS spectrum. The resulting error for the $\mathrm{BrO} S C$ will then be significantly larger.

It should be noted that no significant differences could be found computing $I^{ \pm \text {VRS }}$ with or without taking into account RRS. A direct comparison of $r_{w}$ shows marginal differences. A DOAS retrieval of $\mathrm{BrO}$ using both types of VRS compensation spectra based on model data led to maximum errors in the retrieved slant column of less than $0.5 \%$. The interaction of atmospheric RRS and oceanic VRS depends on a number of parameters. However, for most of the realistic GOME scenarios no significant error is expected. This has important consequences for computational speed, as model runs accounting for VRS but excluding RRS are significantly faster.

\subsection{Retrievals of other gases}

Similar to the model retrieval of $\mathrm{BrO}$ several tests have been made fitting $\mathrm{HCHO}$. All quantities used in the fit of $\mathrm{HCHO}$ were modeled using SCIATRAN for a wavelength window slightly larger than the fitting window of 337.35-356.12 nm that is used for HCHO retrieval from GOME (Wittrock et al., 2000).

The results are comparable to those of $\mathrm{BrO}$. However, neglecting VRS results in larger errors for $\mathrm{HCHO}$ than in the case of $\mathrm{BrO}$.

The omission of a Ring spectrum leads to the worst fit results. However, the introduction of wrong RRS and VRS spectra led to smaller errors than in the case of $\mathrm{BrO}$ retrievals. Wrong model assumptions for VRS were not provoking as large errors as the same poor assumptions in modeling of RRS.

VRS' impact on DOAS retrievals of $\mathrm{O}_{3}$ and $\mathrm{SO}_{2}$ are expected to be negligible due to spectrally unstructured contributions from this effect in the wavelength range between $300-340 \mathrm{~nm}$. 

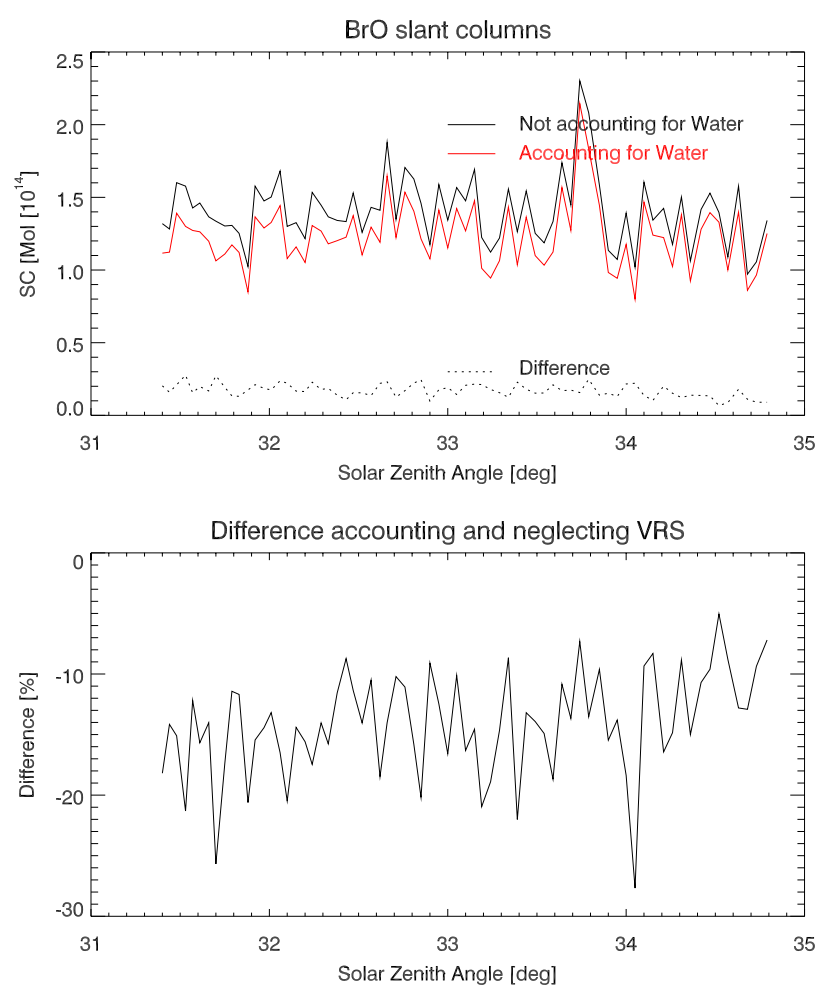

Fig. 5. DOAS fits of clear-sky GOME data (Level 1 filename: 90208175).

Similar behavior will usually not have to be expected for $\mathrm{OClO}$ even though it is commonly retrieved in the critical UV wavelength region. The gas is evaluated only at high solar zenith angles and mostly in polar regions where chlorophyll levels tend to be higher and therefore the impact of VRS is not expected to be large. It is however relevant if $\mathrm{OClO}$ analysis is performed at low latitudes.

\section{BrO retrieval from GOME}

In order to demonstrate the magnitude of the impact (not the error!) on measured data exemplarily, we have modeled a VRS spectrum for a SZA of $33^{\circ}$ and included it in a DOAS fit on a series of consecutive GOME pixels located over oligotrophic ocean regions.

Figure 5 shows the results for clear-sky measurements from 1999 (orbit 19892). BrO was used as the target species. Reference spectra for $\mathrm{NO}_{2}, \mathrm{O}_{4}$ and $\mathrm{O}_{3}$ for two temperatures were fitted together with a modeled Ring and VRS compensation spectrum at $33^{\circ} \mathrm{SZA}$, and an under-sampling correction spectrum. A shift-and-squeeze of the wavelength axis of each spectrum relative to the GOME irradiance has been performed as part of the DOAS fit. $r_{r}$ and $r_{w}$ were computed with an adequate solar irradiance input from GOME.

In the upper plot $\mathrm{BrO} S C$ s are shown for the two cases, with (red) and without (black) VRS correction in the DOAS fit. The $S C$ were plotted as a function of SZA (i.e. geolocation). The lower plot shows the relative difference between both results (Diff $=100.0 *\left(S C_{\text {WithoutVRS }}-\right.$ $\left.\left.S C_{\text {WithVRS }}\right) / S C_{\text {WithVRS }}\right)$. Differences between more than $+20 \%$ and $-5 \%$ can be observed. These values fit to the range of theoretical ones shown in Table 1 but tend to be lower, suggesting a higher chlorophyll concentration and therefore less impact on the OD. Still, there are uncertainties on the impact of inaccurate absorption spectra of water in this wavelength region. An over-weighting of the impact of VRS on the retrievals might be the case.

The discrepancy between model and experimental data has still to be analyzed and is possibly related to inaccurately modeled inherent optical properties of sea water. A possible reason for the discrepancy could also be the compensation of VRS spectral structures through "shifting \& squeezing" (relative wavelength calibration), as well as under-sampling correction spectra (especially relevant for GOME retrievals in the UV).

\section{Conclusions}

A simple ocean reflectance model introducing Vibrational Raman Scattering (VRS) as proposed by Sathyendranath and Platt (1998) and Vasilkov et al. (2002b) has been incorporated in the atmospheric radiative transfer model SCIATRAN.

Model runs have been performed and the results have been qualitatively compared to results of another model. Good agreement could be found. The validation of the model against GOME data and those of dedicated measurements of the upwelling radiance above clear swimming pool water showed good results demonstrating that the model provides an adequate simulation of the effect.

A scheme was developed that is capable to compensate the impact of VRS on DOAS retrievals (similar to the compensation of the Ring effect) using a simple VRS compensation spectrum to be used as a pseudo absorber in the fit. This scheme is able to replace empirical approaches formerly used to reduce spectral signatures of VRS in DOAS retrievals.

In a sensitivity study, optical depths evaluated with model reference spectra showed promising results using a VRS compensation spectrum in order to correct for the impact of VRS. However, neglecting the effect led to large errors in the Slant Column $(S C)$ of Bromine Monoxide, $\mathrm{BrO}$ and potentially also $\mathrm{HCHO}$.

However, either an over-estimation of the impact of VRS (due to potentially erroneous inherent optical properties) has been modeled or the impact of VRS on the retrieval of both gases from experimental data is compensated by shift \& squeeze algorithms and under-sampling correction spectra.

The introduction of a compensation spectrum even if it is modeled under wrong assumptions yields significantly smaller errors in the model tests for both investigated gases. 
This makes the VRS compensation spectrum a promising and simple tool to optimize the GOME DOAS fits at low latitudes.

A similar impact can be expected also for forthcoming missions like GOME-2 and OMI, as well as for the already deployed instrument SCIAMACHY.

Future work comprises the introduction of improved input parameters for the reflectance model and a feasibility study to retrieve chlorophyll-a concentrations using the VRS compensation spectrum with DOAS.

Acknowledgement. The authors would like to thank the SeaWiFS Project and the Goddard Earth Sciences Data and Information Services Center/Distributed Active Archive Center for the distribution of the SeaWiFS data. We thank both anonymous Referees for helpful comments. Also, we would like to thank V. V. Rozanov and $\mathrm{R}$. de Beek for stimulating discussions and critical reading of the manuscript. M. Vountas would like to thank A. Vountas for inspiration.

\section{References}

Bartlett, J., Voss, K., Sathyendranath, S., and Vodacek, A.: Raman scattering by pure water and seawater, Appl. Opt., 37, 1998.

Brühl, C. and Crutzen, P.: Chemo-dynamical model of the atmosphere: Profile data base, personal communication, 1992.

Burrows, J., Eisinger, M., and Vountas, M.: Design, Development and Upgrading of Prototype Software Tools for Use with ESRIN Atmopheric Reference Processor, Tech. rep., ESRIN Report, ESRIN Contract 12030/96/I-H-GE, 1998.

Burrows, J. P., Weber, M., Buchwitz, M., Rozanov, V., LadstätterWeißenmayer, A., Richter, A., de Beek, R., Hoogen, R., Bramstedt, K., Eichmann, K.-U., Eisinger, M., and Perner, D.: The Global Ozone Monitoring Experiment (GOME): Mission concept and first scientific results, J. Atmos. Sci., 56, 151-175, 1999.

de Beek, R., Vountas, M., Rozanov, V., Richter, A., and Burrows, J.: The Ring effect in the cloudy atmosphere, Geophys. Res. Let., 28, 2001 .

Ge, Y., Gordon, H., and Voss, K.: Simulation of inelastic-scattering contributions to the irradiance field in the ocean: variation in Fraunhofer line depths, Appl. Opt., 32, 1993.

Grainger, J. and Ring, J.: Anomalous Fraunhofer line profiles, Nature, 193, 762, 1962.

Haltrin, V. and Kattawar, G.: Self-consistent solution to the equation of radiative transfer with elastic and inelastic scattering in ocean optics: 1 model, Appl. Opt., 32, 1993.

Kattawar, G. and Xu, X.: Filling in of Fraunhofer lines in the ocean by Raman scattering, Appl. Opt., 30, 1992.

Kattawar, G., Young, A., and Humphreys, T.: Inelastic scattering in planetary atmospheres, I, the Ring effect, without aerosols, Astrophys. J., 243, 1049-1057, 1981.

Kneizys, F., Shettle, E., Abreu, L., Chetwynd, J., Anderson, G., Gellery, W., Selby, J., and Clough, S.: Users Guide to LOWTRAN 7, Tech. Rep. AFGL-TR-86-0177, Air Force Geophys. Lab., Hanscom AFB, Mass., 1986.

Kurucz, R., Furenlid, I., Brault, J., and Testerman, L.: Solar flux atlas from 296 to $1300 \mathrm{~nm}$, Tech. rep., National Solar Observatory, Sunspot, New Mexico, 1984.
Marshall, B. and Smith, R.: Raman scattering and in-water ocean optical properties, Appl. Opt., 29, 1990.

Mobley, C.: Light and Water-Radiative Transfer in Natural Waters, Academic Press, 1994.

Morel, A.: Optical modeling of the upper ocean in relation to its biogeneous matter content (case i waters), J. Geophys. Res., 93, 1988.

Morel, A. and Prieur, L.: Analysis of variations in ocean color, Limnol. Oceanogr., 22, 1977.

Patterson, K. W.: Contribution of chromophoric dissolved organic matter to attenuation of UV radiation in three contrasting coastal areas, PHD work, UCLA, Santa Barbara, 2000.

Platt, U. and Perner, D.: Direct measurement of atmospheric $\mathrm{HCHO}, \mathrm{HNO}_{2}, \mathrm{O}_{3}, \mathrm{NO}_{2}$ and $\mathrm{SO}_{2}$ by differential optical absorption spectroscopy, J. Geophys. Res., 85, 1980.

Pope, R. and Fry, E.: Absorption spectrum (380-700 nm) of pure water. ii. integrating cavity measurements, Appl. Opt., 36, 1997.

Quickenden, T. and Irvin, J.: The ultraviolet absorption spectrum of liquid water, J. Chem. Phys., 72, 1980.

Richter, A. and Burrows, J.: A multi wavelength approach for the retrieval of tropospheric $\mathrm{NO}_{2}$ from GOME measurements, in proceedings of the ERS-ENVISAT symposium, Gothenburg October 2000, ESA publication SP-461, 2000.

Richter, A., Wittrock, F., Eisinger, M., and Burrows, J. P.: GOME observations of tropospheric $\mathrm{BrO}$ in northern hemispheric spring and summer 1997, Geophys. Res. Lett., 25, 2683-2686, 1998.

Rozanov, V., Diebel, D., Spurr, R., and Burrows, J.: GOMETRAN: A radiative transfer model for the satellite project GOME - the plane-parallel version, J. Geophys. Res., 1997.

Sathyendranath, S. and Platt, T.: Ocean-color model incorporating transspectral processes, Appl. Opt., 37, 1998.

Smith, R. and Baker, K.: Optical properties of the clearest natural waters (200-800nm), Appl. Opt., 20, 1981.

Solomon, S., Schmeltekopf, A. L., and Sanders, R. W.: On the interpretation of zenith sky absorption measurements, J. Geophys. Res., 92, 8311-8319, 1987.

Stavn, R. and Weidemann, A.: Optical modeling of clear ocean light fields: Raman scattering effects, Appl. Opt., 27, 1988.

Vasilkov, A., Herman, J., Krotkov, N., Kahru, M., Mitchell, B., and Hsu, C.: Problems in assement of the ultraviolet penetration into natural waters from space-based measurements, Opt. Eng., 41, 2002a.

Vasilkov, A. P., Joiner, J., Gleason, J., and Bhartia, P.: Ocean Raman scattering in satellite backscatter UV measurements, Geophys. Res. Let., 10, 2002b.

Vountas, M.: Modeling and Parameterization of the Ring Effect: Impact on the retrieval of stratospheric Trace Gases-PHD work In German, ISBN 3-8265-4649-0, Shaker, 1998.

Vountas, M., Rozanov, V., and Burrows, J.: Ring effect: Impact of rotational Raman scattering on radiative transfer in earth's atmosphere, J. Quant. Spectrosc. Radiat. Transfer, 60, 943-961, 1998.

Walrafen, G.: Raman spectral studies of the effects of temperature on water structures, J. Chem. Phys., 47, 1967.

Wittrock, F., Richter, A., Ladstätter-Weißenmayer, A., and Burrows, J. P.: Global observations of formaldehyde, in proceedings of the ERS-ENVISAT symposium, Gothenburg October 2000, ESA publication SP-461, 2000.

Young, A.: Rayleigh scattering, Phys. Today, 1982. 